MATEC Web of Conferences 48,04002 (2016)

DOI: $10.1051 /$ matecconf $/ 20164804002$

C) Owned by the authors, published by EDP Sciences, 2016

\title{
A Single Information Space for aerospace enterprises based on a Service Oriented Architecture
}

\author{
Ekaterina Vasilyeva ${ }^{1, a}$ and Nikolay Markov ${ }^{1}$ \\ ${ }^{1}$ National Research Tomsk Polytechnic University, 634050 Tomsk, Russia
}

\begin{abstract}
Nowadays aerospace enterprises have a variety of Information Systems (IS) and Software Complexes (SC). Their strategic aim is to integrate all IS and SC, thus to create the Single Information Space (SIS). An approach to create the SIS based on a Service Oriented Architecture (SOA) concept at aerospace enterprises is suggested. It was shown that different tools to implement this approach are available. The SOA model of the SIS of the typical enterprise, which has a custom set of services, IS and SC, was developed. Two modifications of this SOA model are proposed. The first modification is suitable for creation of the SIS on enterprises which have Business Intelligence (BI) systems based on Data Warehouse (DW). Another modification of the SOA-based SIS is important for those enterprises which require to process at least the part of technological and manufacturing data in a real-time mode.
\end{abstract}

\section{Introduction}

Nowadays manufacturing enterprises of aeronautical and space branches (hereinafter called as aerospace) are characterized by a huge amount and diversity of issues to solve. Thus, there are many executable Business Processes (BP) which need to be automated. The situation is complicated by several management levels (from a manufacturing sector, a workshop to a management company) which interact in such enterprises. Hence the number of new IS which are implemented to automate routine actions of workers and to support a decision-making on each management level is constantly increasing. Also enterprises use Automated Process Control Systems (APCS) to manage technological processes. Many enterprises have various Laboratory Information Management Systems (LIMS).

Some of Russian aerospace enterprises use or implement Corporate Information Systems (CIS) which are able to automate a work of some enterprise's services and BP. Usually, CIS is presented by a system that controls all enterprise's resources - Enterprise Resource Planning (ERP). However, the experience [1] has shown that ERP system does not allow automating technological processes and some manufacturing processes of the industrial enterprise, so enterprises use a variety of APCS and a system for the manufacturer executive managing which known as Manufacturing Execution System (MES) [2] in addition to ERP system. Some enterprises have not the developed system of ERP class but have an implemented multifunctional MES to control many of enterprise's services, and this MES can act as CIS. Some characteristics of aerospace enterprises (high-tech manufacturing that can be changed in a short time period to release new products; production of unique products or products in small quantities, etc.) determine the necessity to keep on use specialized IS and SC in addition to

\footnotetext{
${ }^{a}$ Corresponding author : katya-vas@mail.ru
} 
mentioned systems. In most cases such IS and SC are presented by Product Lifecycle Management (PLM) systems, Supply Chain Management (SCM) systems and etc. The large equipment stock, various test stands, unique systems for enterprise's technological tooling at such enterprises leads to necessity of implementation IS to control the technical maintenance and repair of these items of equipment - Maintenance, Repair, and Operations (MRO) system.

The strategic aim of the enterprise which has a large amount of various IS and SC is to create a SIS [3]. The SIS is a set of embedded in the enterprise IS and SC which are interrelated and work according to a common base of principles and rules, providing a convenient interaction between all enterprise's services and employees with a full satisfaction of their information requests. The SIS allows avoiding duplication of input data, to create and use common for whole enterprise classifiers, dictionaries and standards and other similar facilities.

Various methods of integration of IS and SC can be used to create the SIS: data integration, functional services integration and etc. [3]. Nowadays the problem of the creation of the SIS in most cases is solved by data integration with ability to exchange the data between two or more specific IS by using partial integration solutions ("point-to-point" integration). This approach has a number of drawbacks associated with the lack of flexibility and scalability of these solutions. A more general approach to solve the problem at the enterprise level is to use hubs or integration platforms $[2,3]$. The most famous modern multifunctional integration platforms are IBM Web Sphere and Microsoft Biz Talk [4]. Along with the versatility and flexibility of these platforms, they are ineffective because of their architecture complexity and the absence of the orientation on specific industries. That's why in recent years industry-based platforms have been developed, for example, for enterprises producing oil and gas $[2,5]$. Unfortunately, there are not such specialized platforms in aerospace enterprises.

There is an idea of integration of IS with different architectures and functionality by using a SOA concept in [6], which was succeed. This article deals with the development of this idea, suggested to create the SIS based on SOA concept at aerospace enterprises. Moreover, the SIS may include both existing IS and SC and newly developed and implemented at the enterprise IS and SC, which are presented by a set of services (web services). Tools for creation of the SIS are considered and the SIS SOA model is proposed.

\section{Features of creation of the SOA-based SIS for enterprises}

We consider the approach to the creation of the SOA-based SIS for manufacturing enterprises, which takes into account the specific requirements for the SIS in the aerospace industry. The basic requirements are:

- to reduce the cost of integration of existing enterprise's IS and SC;

- to reduce the time and cost of development and integration of new IS and software modules into the SIS;

- to increase the speed of applying changes, the list of components of the SIS and the flexibility of $\mathrm{BP}$ in the case of rapidly changing business requirements in the aerospace industry (for example, a change in the product mix or the implementation of new technologies).

We show how these requirements can be met through the creation of SIS based on a concept of SOA. SOA is a modular approach to the development of software and IS that based on the use of distributed, loosely coupled and replaceable software components (services), which are equipped with standardized interfaces for communication by standardized protocols [6]. In this case the service is standalone software component (module) with the possibility of self-description which performs a specific business task (BP, sub process, function) and provides some of the functionality by the request to IS or other services. In most cases web services are developed. Loosely coupled services provide a simple and fast adaptation of the SIS to changes in enterprise's BP. The services can work standalone or to be combined with other services to create a new composite applications (IS or SC). The services allow implementing a particular BP by their interaction on the network in a certain sequence. The service should be used according to the convention of the interface. The interfaces are used for providing the service's methods to the outside world and to organize an interaction of 
services. The service interface contains the description of input parameters and of the result of its work.

Another key component of SOA is a service registry. It is presented by a catalogue and includes descriptions of services (their physical location, versions and validity period; documentation for services). Due to using the service registry SOA has loosely coupled services. The third component of SOA is an Enterprise Service Bus (ESB) [7]. The ESB is connective software for all existing (legacy) IS and SC on the enterprise and all new developed services to provide centralized and unified eventdriven messaging between IS, SC and new enterprise's services. All settings for the processing and sending of messages are usually concentrated in a single point of ESB. It means that in case of change of any IS, SC or service connected to the bus there is no need to reconfigure other systems and services of the SIS. The ESB is responsible for a number of classical tasks of integration of IS, SC and services:

- message routing;

- conversion of message formats and protocols;

- transaction support;

- providing security, auditing, logging.

Finally, another component of the SOA is Business Process Management (BPM) system. BPM system is software providing opportunities to start, coordinate, manage, administrate and debug the $\mathrm{BP}$. The BP is deemed to be a sequence of logically related actions that transform input data into results or output data. In other words, BP determines a startup sequence for IS, SC and services to solve specific business task. Management of the startup sequence is called orchestration. BPM system also controls the choreography in the case of the exchange of messages between IS, SC and services.

Summarizing the brief analysis of the components of SOA and its main features, we can assume that SOA is a promising model of interaction between the SIS components (legacy IS, SC and new services). This model allows the components to communicate with each other through specified interfaces. The interfaces are not dependent on hardware platforms, operating systems and programming languages that were used to develop components of the SIS on the enterprise. This allows components to communicate with each other by the same standard and versatile way. This aspect of using interfaces that are independent of the environment and the platform is called "loose coupling" model. This model provides greater flexibility and adaptability of the SIS in the changing requirements of the enterprise as the replacement or upgrading a component of the SIS will not affect the others. Therefore, the SOA-based SIS will meet the basic requirements for the SIS in the aerospace.

\section{Tools for implementation the SOA-based SIS}

We made a brief overview of tools and technologies to create the SOA-based SIS on the enterprise. Following two most common technologies are used to describe the functions of services and messages [7]:

- Web Services Description Language (WSDL) is a language for web-services and access to them; the technology of development of such services divides the description of services into the description of the interface and shell: the interface describes what must contain the request and the shell determines the data and transport protocols;

- Simple Object Access Protocol (SOAP) is a protocol for exchange structured messages that is used for description of format of received and sent messages.

The ESB should be presented by one of service buses of known software companies such as Oracle, IBM, Microsoft and etc. More full ESB functionality is presented in Oracle ESB, Open ESB and IBM Web Sphere ESB [7]. Most ESB comes with a large number of pre-configured adapters: adapters for ERP systems (SAP R/3, Oracle eBusiness Suite, 1C, MS Ax 2009), adapters for databases and etc.

The BPM-system describes the coordination of BP using the Business Process Execution Language (BPEL). The description of BP on BPEL is compiled into an executable code of the BPM 
system that allows to recover described BP from available IS, SC and services [8]. In recent years a more advanced language for modeling BP was invented - the language BPMN (Business Process Model and Notation) [9]. BPMN specification allows to present BP as diagrams and to convert them to BPEL. There is an example of such diagram on the figure 1 which describes the BP "Inspection of workshop's production equipment". Sub processes "Forming an inspection list", "Filling of electronic diagnostics journals" and "Filling of electronic operations journals" are executed in the MRO system, sub process "Informing of related units" can be implemented in Electronic Document Management System (EDMS), sub process "Responding to a failure" can be implemented by MES with creation of the task to do unscheduled work and making orders of required material resources in the ERP system. The BPM system which is based on the diagram is aimed to coordinate all of the mentioned IS. The BPM system can be presented by widely known systems: Orchestra (supports both languages), Bonita BPM (language supports BPMN), etc.

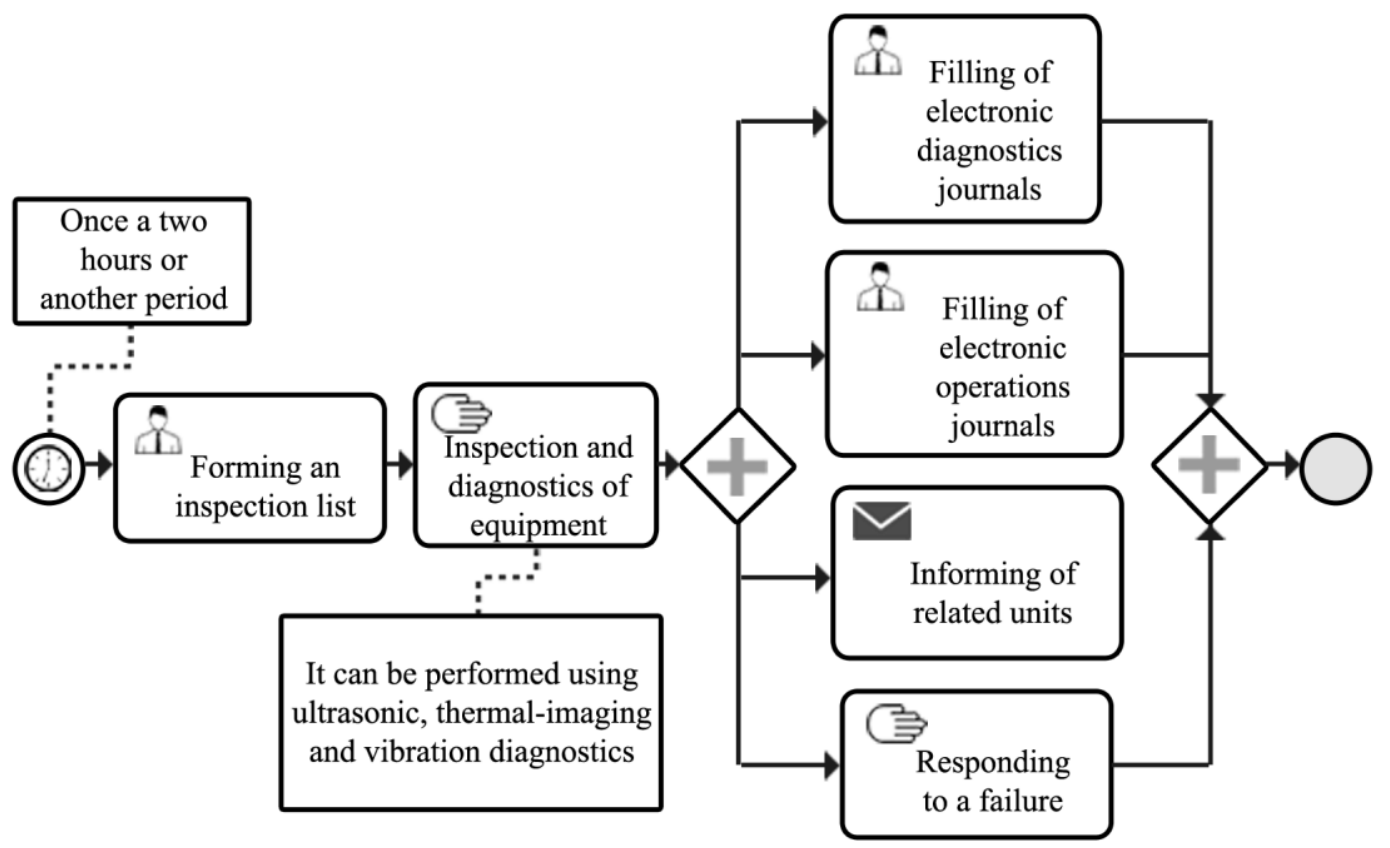

Figure 1. BPMN diagram of the BP "Inspection of workshop's production equipment”.

\section{SOA models of the SIS of enterprises}

The approach to the creation of the SOA-based SIS on aerospace enterprises is described above. We reviewed features of it in context of the typical aerospace enterprise which has standard set of IS, SC and services. The SOA model is based on ESB (figure 2) which usually presented by one of described buses. The most of legacy IS and SC (PLM system, ERP system, SCM system, MES, EDMS, MRO system and etc.) have adapters to communicate by the ESB. Some adapters are standard and they are supplied with the ESB, while others should be developed according to the specification of the concrete IS. Special OPC adapters (based on the open standard OLE for Process Control) are implemented into all enterprise's APCS and LIMS to provide an access to technological data and results of laboratory research through some IS. Widely used specifications OPC DA (Data Access) and OPC XML DA allow real-time exchanging data with programmable logic controllers, numeric control and etc. Typical enterprises also must have a system to control referenced data (SCRD). Using the SOA model 
has some benefits, for example, BPM system is able to perform EDMS functions. Services (from 1 to $\mathrm{N})$ for new IS and SC are created accordingly their interface specifications.

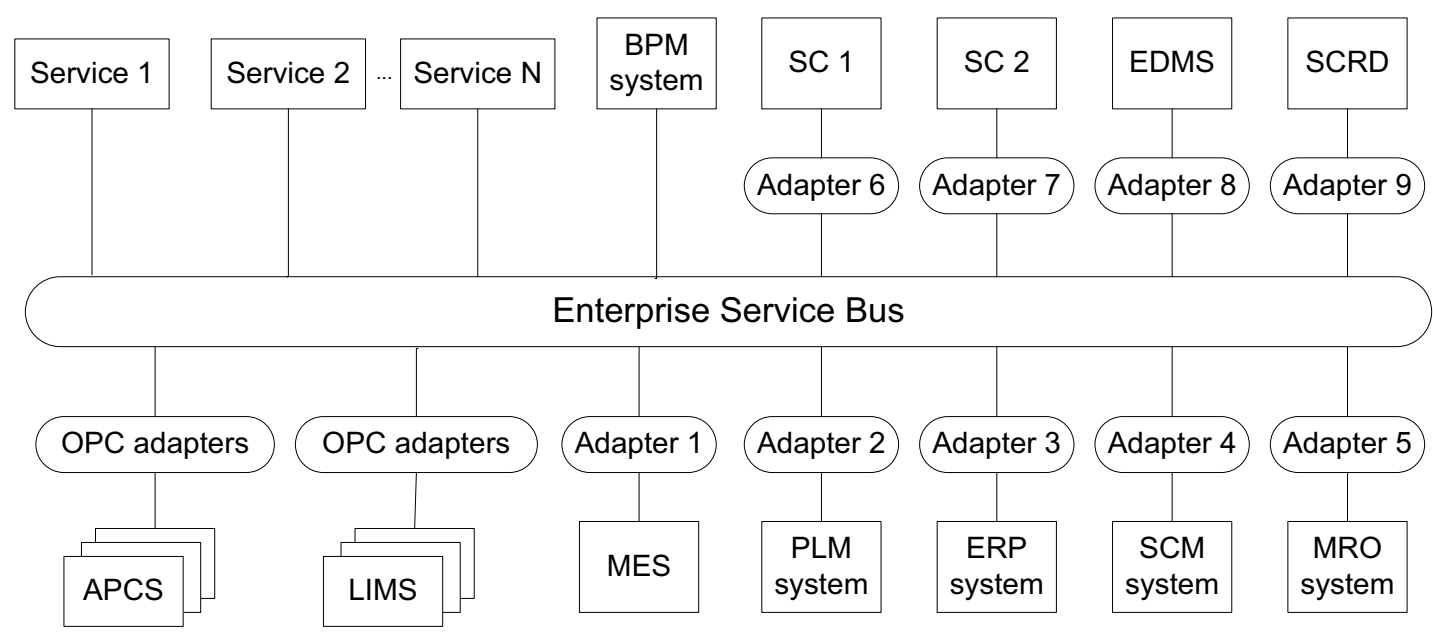

Figure 2. The SIS architecture of the typical enterprise.

The SIS of untypical aerospace enterprise additionally can contain BI systems based on DW. Moreover, in case of BI analysis of big data from APCS, LIMS and MES the SOA model of the SIS of the typical enterprise will be complicated by additional system bus for BI systems and DW. The additional bus provides the data and messages exchange between BI systems and DW that allows decreasing the usage of the ESB.

The SOA model of the SIS of the typical enterprise should be modified by additional Manufacturing Services Bus (MSB) to provide an opportunity of real-time technological data processing (according to the concept "Manufacture 2.0" [7]). In this case SOA model from the figure 2 should be integrated with manufacturing SOA model and some IS and SC will be moved to the MSB. It allows organizing the technological and manufacturing data and messages exchange in a realtime mode by facilities of the manufacturing SOA model.

\section{Conclusion}

Nowadays aerospace manufacturing enterprises are characterized by a huge amount of IS and SC to automate a variety of BP. At the same time there is a necessity to create the SIS that will provide a convenient interaction between all enterprise's services and employees when they interact with IS and SC. The SIS is a set of embedded in the enterprise IS and SC which are interrelated and work according to a common base of principles and rules. The SIS allows avoiding duplication of input data, to create and use common for whole enterprise classifiers, dictionaries and standards and other similar facilities.

The approach of creating the SIS based on SOA, which is able to meet requirements for the SIS of aerospace enterprises, is presented in the article. One of such requirements is a flexibility of BP in the case of rapidly changing business requirements in the aerospace industry, and the main requirement for the automation process of BP is a high speed of applying changes to the SIS components. It is shown that creation of the SOA-based SIS will provide the required for aerospace enterprises flexibility in the case of implementation of new services or removing legacy IS and SC. This can be achieved through the organization of services, IS and SC interaction through the ESB. An analysis of tools and technologies for development of SOA models of the SIS has shown that all necessary tools for this are available. The ESB can be presented by one of service buses of known software companies. It is suggested to use one of widely known BPM systems which support BPEL and (or) BPMN. 


\section{MATEC Web of Conferences}

The SOA model of the SIS of the typical enterprise, which has a custom set of services, IS and SC, was developed. This SOA model can be modified according to changing requirements of the enterprise. The modification for enterprises which have BI systems based on DW is presented. This modification has an additional bus for BI systems and DW which is integrated with the ESB. The additional bus provides the data and messages exchange between BI systems and DW that allows decreasing the usage of the ESB. Another modification of the SOA-based SIS is important for those enterprises which require to process at least the part of technological and manufacturing data in a realtime mode. In this case the SOA model of the SIS of the typical enterprise integrates with the manufacturing SOA model which has the MSB. Using the manufacturing SOA model supposes moving IS, which are working in a real-time mode (APCS, LIMS), to the MSB. It allows organizing the technological and manufacturing data and messages exchange in a real-time mode by facilities of the manufacturing SOA model.

\section{References}

1. J. Kletti (Ed.) Manufacturing Execution Systems - MES, (Berlin Heidelberg: Springer-Verlag, 2007)

2. A. V. Kudinov, N. G. Markov, Problemy avtomatizacii proizvodstva gazodobyvajushhih kompanij (Tomsk: izdatelstvo Tomskogo politehnicheskogo universiteta, 2012)

3. N. G. Markov, Informacionnoe obshhestvo 3, 53 (2014)

4. Microsoft BIZ Talk Server. URL: https://www.microsoft.com/en-us/servercloud/products/biztalk/ (2016)

5. V. Veyver, A. Kudinov, N. Markov, International Journal of Computer Applications 5, 14 (2012)

6. M. Juric, SOA approach to integration (Birmingham: Packt Publishing Ltd, 2007)

7. A. Boyd, D. Noller, P. Peters, WP 27. SOA in Manufacturing Guidebook (MESA International, IBM Corporation and Capgemini co-branded white paper, 2010)

8. W. H. Utomo, T. Wellem, JATIT 62, 364 (2014)

9. BPMN - model biznes-processov i notacija. URL: www.elma-bpm.ru (2016) 